\title{
A second-order Fourier pseudospectral method for the generalized regularized long wave equation
}

\author{
Xiaorong Kang ${ }^{1}$, Kelong Cheng ${ }^{1}$ and Chunxiang Guo ${ }^{2 *}$
}

\section{"Correspondence:}

guocx70@163.com

${ }^{2}$ School of Business, Sichuan

University, Chengdu, 610064

P.R. China

Full list of author information is

available at the end of the article (c) 2015 Kang et al. This article is distributed under the terms of the Creative Commons Attribution 4.0 International License (http://creativecommons.org/licenses/by/4.0/), which permits unrestricted use, distribution, and reproduction in any medium, provided you give appropriate credit to the original author(s) and the source, provide a link to the Creative Commons license, and indicate if changes were made.

where $u_{0}(x)$ is the given known function, $p \geq 2$ is an integer and $\alpha, \beta$ are constants. The RLW equation $(p=2)$ is originally introduced to describe the behavior of the undular bore by Peregrine [1] and plays a major role in the study of nonlinear dispersive waves, such as shallow water waves and ion acoustic plasma waves. Mathematical theory for this equation was presented in $[2,3]$ and the references therein. Due to nonlinearity of the GRLW equation, only a few exact solutions exist [4]. Therefore, the higher accurate numerical methods are essential for capturing physical phenomena accurately. Many efforts have been made to develop numerical method for solving this equation, such as the variational iteration method [5], the finite difference method [6-10], the Fourier pseudospectral method [11, 12], the Galerkin element method [13, 14], the Adomian decomposition method [15, 16], the collocation method $[17,18]$, and others. 
In addition to the nonlinearity, another interesting feature of the GRLW equation is that it has both stable and unstable solitary-wave solutions when $p \geq 5$ [19]. Bona et al. [20] proposed a high-order accurate pseudospectral scheme to approximate the solutions of the GRLW equation and to examine the dynamics of the suitably perturbed solitary waves. In their experiments, they found that unstable solitary waves may evolve into several, stable solitary waves, and the positive initial data does not need the feature of the solitary waves for the long-time asymptotic behavior. Also, similar discussions for the generalized Benjamin-Bona-Mahony equation can be found in [21].

On the other hand, spectral and pseudospectral methods have been developed rapidly in the past two decades. They have been applied to numerical simulations in many fields because of its high accuracy. For instance, the stability analysis for linear time-dependent problems can be found in $[22,23]$. Some pioneering works for nonlinear equations were initiated by Maday and Quarteroni [24] for steady-state spectral solutions. Also note the analysis of one-dimensional conservation laws by Tadmor [25, 26], semi-discrete viscous Burgers' equation and Navier-Stokes equations by Weinan [27], Fourier spectral projection method for Navier-Stokes equations by Guo and Zou [28], fully discrete (discrete both in space and time) pseudospectral method applied to viscous Burgers' equation and Boussinesq equation in $[29,30]$ by Gottlieb and Wang, and Fourier spectral approximation to the flow of liquid crystals [31] by Du et al., and so forth.

In this paper, we present a fully discrete semi-implicit Fourier pseudospectral method for the GRLW equation, with periodic boundary condition on $\left[x_{L}, x_{R}\right]$. This periodicity assumption is reasonable if the solution decays exponentially outside $\left[x_{L}, x_{R}\right]$ for large enough $x_{L}$ and $x_{R}$. For the theoretical analysis, the convergence analysis follows the combination of consistency analysis for the proposed scheme and the stability analysis for the numerical error function. The consistency analysis shows that such an approximate solution satisfies the numerical scheme with an $O\left(\Delta t^{2}\right)$ accuracy in time and a spectral accuracy in space. Based on the a priori estimate, a detailed error estimate is performed by an aliasing error control estimate for the nonlinear terms, and the $a$ priori assumption can be recovered. Additionally, various numerical experiments are given to verify the theoretical analysis, wave interaction and evolution of the unstable solitary wave, respectively.

The remainder of the paper is organized as follows. In Section 2, we review the Fourier pseudospectral method and present a second-order in time linearized semi-implicit Fourier pseudospectral scheme for the GRLW equation. In Section 3, the consistency analysis is discussed in detail, and the stability and convergence analysis are reported in Section 4. Finally, we present we present many numerical simulations related to different types of the GRLW equations in Section 5.

\section{Numerical scheme}

\subsection{Reviews of pseudospectral approximation}

For $f(x) \in L^{2}(\Omega), \Omega=(0, L)$, with Fourier series

$$
f(x)=\sum_{l=-\infty}^{\infty} \hat{f}_{l} \mathrm{e}^{2 \pi \mathrm{i} l x / L}, \quad \text { with } \hat{f}_{l}=\int_{\Omega} f(x) \mathrm{e}^{-2 \pi \mathrm{i} l x / L} d x
$$


its truncated series is defined as the projection onto the space $\mathcal{B}^{N}$ of trigonometric polynomials in $x$ of degree up to $N$, given by

$$
\mathcal{P}_{N} f(x)=\sum_{l=-N}^{N} \hat{f}_{l} \mathrm{e}^{2 \pi \mathrm{i} l x / L} .
$$

To obtain a pseudospectral approximation at a given set of points, an interpolation operator $\mathcal{I}_{N}$ is introduced. Given a uniform numerical grid with $(2 N+1)$ points and a discrete vector function $\mathbf{f}$ where $\mathbf{f}_{i}=f\left(x_{i}\right)$, for each spatial point $x_{i}$. The Fourier interpolation of the function is defined by

$$
\left(\mathcal{I}_{N} f\right)(x)=\sum_{l=-N}^{N}\left(\hat{f}_{c}^{N}\right)_{l} \mathrm{e}^{2 \pi \mathrm{i} l x / L}
$$

where the $(2 N+1)$ pseudospectral coefficients $\left(\hat{f}_{c}^{N}\right)_{l}$ are computed based on the interpolation condition $f\left(x_{i}\right)=\left(\mathcal{I}_{N} f\right)\left(x_{i}\right)$ on the $2 N+1$ equidistant points. These collocation coefficients can be efficiently computed using the fast Fourier transform (FFT). Note that the pseudospectral coefficients are not equal to the actual Fourier coefficients; the difference between them is known as the aliasing error. In general, $\mathcal{P}_{N} f(x) \neq \mathcal{I}_{N} f(x)$, and even $\mathcal{P}_{N} f\left(x_{i}\right) \neq \mathcal{I}_{N} f\left(x_{i}\right)$, except of course in the case that $f \in \mathcal{B}^{N}$.

The Fourier series and the formulas for its projection and interpolation allow one to easily take derivative by simply multiplying the appropriate Fourier coefficients $\left(\hat{f}_{c}^{N}\right)_{l}$ by $2 l \pi \mathrm{i} / L$. Furthermore, we can take subsequent derivatives in the same way, so that differentiation in physical space is accomplished via multiplication in Fourier space. As long as $f$ and all is derivatives (up to $m$ th order) are continuous and periodic on $\Omega$, the convergence of the derivatives of the projection and interpolation is given by

$$
\begin{aligned}
& \left\|\partial^{k} f(x)-\partial^{k} \mathcal{P}_{N} f(x)\right\|_{L^{2}} \leq C\left\|f^{(m)}\right\|_{L^{2}} h^{m-k}, \quad \text { for } 0 \leq k \leq m, \\
& \left\|\partial^{k} f(x)-\partial^{k} \mathcal{I}_{N} f(x)\right\|_{L^{2}} \leq C\|f\|_{H^{m}} h^{m-k}, \quad \text { for } 0 \leq k \leq m, m>\frac{d}{2},
\end{aligned}
$$

where $d$ denotes the space dimension, $\|f\|_{L^{2}}=\left(\int_{\Omega} f^{2}(x) d x\right)^{1 / 2}$, and

$$
\|f\|_{H^{m}}=\left(\int_{\Omega} \sum_{|\alpha| \leq m}\left|\left(D^{\alpha} f\right)^{2}(x)\right| d x\right)^{1 / 2} .
$$

For more details, see the discussion of approximation theory by Canuto and Quarteroni [32].

For any collocation approximation to the function $f(x)$ at the points $x_{i}$

$$
f\left(x_{i}\right)=\left(\mathcal{I}_{N} f\right)_{i}=\sum_{l=-N}^{N}\left(\hat{f}_{c}^{N}\right)_{l} \mathrm{e}^{2 \pi \mathrm{i} l x_{i} / L},
$$

one can define discrete differentiation operator $\mathcal{D}_{N}$ operating on the vector of grid values $\mathbf{f}=f\left(x_{i}\right)$. In practice, one may compute the collocation coefficients $\left(f_{c}^{\hat{N}}\right)_{l}$ via FFT, and then multiply them by the correct values (given by $2 l \pi \mathrm{i} / L$ ) and perform the inverse FFT. 
Alternatively, we can view the differentiation operator $\mathcal{D}_{N}$ as a matrix, and the above process can be seen as a matrix-vector multiplication. The same process is performed for the second $\partial_{x}^{2}$, where the collocation coefficients are multiplied by $\left(-4 \pi^{2} l^{2} / L^{2}\right)$. In turn, the differentiation matrix can be applied for multiple times, i.e., the vector $\mathbf{f}$ is multiplied by $\mathcal{D}_{N}^{2}$.

Since the pseudospectral differentiation is taken at a point-wise level, a discrete $L^{2}$ norm and inner product need to be introduced to facilitate the analysis. Given any periodic grid functions $\mathbf{f}$ and $\mathbf{g}$ (over the numerical grid), we note that these are simply vectors and define the discrete $L^{2}$ inner product and norm

$$
\|\mathbf{f}\|_{2}=\sqrt{\langle\mathbf{f}, \mathbf{f}\rangle}, \quad \text { with }\langle\mathbf{f}, \mathbf{g}\rangle=\frac{1}{2 N+1} \sum_{i=0}^{2 N} \mathbf{f}_{i} \mathbf{g}_{i} .
$$

The following summation by parts (see [29]) will be of use:

$$
\left\langle\mathbf{f}, \mathcal{D}_{N} \mathbf{g}\right\rangle=-\left\langle\mathcal{D}_{N} \mathbf{f}, \mathbf{g}\right\rangle, \quad\left\langle\mathbf{f}, \mathcal{D}_{N}^{2} \mathbf{g}\right\rangle=-\left\langle\mathcal{D}_{N} \mathbf{f}, \mathcal{D}_{N} \mathbf{g}\right\rangle
$$

\subsection{Numerical scheme}

Since the standard second-order Adams-Bashforth extrapolation formula involves the numerical solutions at time node points $t^{n}$, $t^{n-1}$, with the well-known coefficients $3 / 2$ and $-1 / 2$, respectively, we propose a linearized semi-implicit Fourier pseudospectral scheme with second-order accuracy in time as follows:

$$
\left(1-\beta \mathcal{D}_{N}^{2}\right) \frac{u^{n+1}-u^{n}}{\Delta t}+\mathcal{D}_{N}\left(\frac{3}{2} u^{n}-\frac{1}{2} u^{n-1}\right)+\alpha \mathcal{D}_{N}\left(\left(\frac{3}{2}\left(u^{n}\right)^{p}-\frac{1}{2}\left(u^{n-1}\right)^{p}\right)\right)=0 .
$$

Remark 2.1 Sloan [11] investigated the RLW equation $(p=2)$ by a three-level explicit Fourier pseudospectral scheme. But numerical tests indicated that the high accuracy in space can only be matched in time with the second-order leap-frog discretization under a strict stability condition. Furthermore, the formal stability and the convergence analysis were not pursued in his work.

Remark 2.2 Although another implicit Fourier pseudospectral scheme discussed by Djidjeli et al. [12] for the GRLW equation can ensure the unconditionally stability, a set of nonlinear algebraic equations has to be solved by a linearizing pseudospectral scheme by the Taylor expansion. Similar to the previous statement, a detailed theoretical analysis also was not given.

\section{Consistency analysis}

Without loss of generality, $C$ denotes a general positive constant, which may have different values in different occurrences. Meanwhile, some function spaces and norms are defined as follows:

$$
\|f\|_{L^{2}\left(0, T: H^{r}\right)}=\left(\int_{0}^{T}\|f\|_{H^{r}}^{2} d t\right)^{\frac{1}{2}}, \quad\|f\|_{L^{\infty}\left(0, T: H^{r}\right)}=\sup _{0 \leq t \leq T}\|f\|_{H^{r}}
$$


and corresponding semi-discrete versions are given by

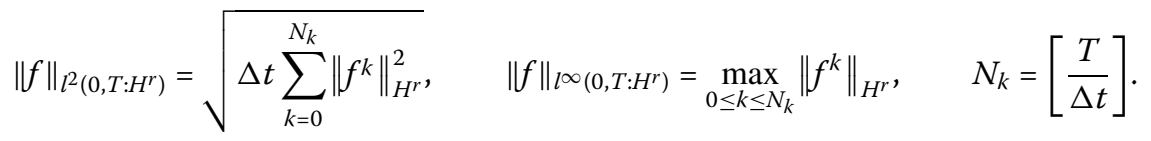

Similarly, other norms used in the next text can be defined and we omit the details.

\subsection{Truncation error analysis for $U_{N}$}

Denote by $u_{e}$ the exact solution and

$$
U_{N}(x, t)=\mathcal{P}_{N} u_{e}(x, t)
$$

From (2.2), the following approximation estimates are clear:

$$
\left\|U_{N}-u_{e}\right\|_{L^{\infty}\left(0, T ; H^{r}\right)} \leq C h^{m}\left\|u_{e}\right\|_{L^{\infty}\left(0, T ; H^{m+r}\right)}, \quad \text { for } r \geq 0,
$$

and

$$
\left\|\partial_{t}^{k}\left(U_{N}-u_{e}\right)\right\|_{H^{r}} \leq C h^{m}\left\|\partial_{t}^{k} u_{e}\right\|_{H^{m+r}}, \quad \text { for } r \geq 0,0 \leq k \leq 2,
$$

which comes from the fact that $\partial_{t}^{k} U_{N}$ is the truncation of $\partial_{t}^{k} u_{e}$ for any $k \geq 0$, since projection and differentiation can commute,

$$
\frac{\partial^{k}}{\partial t^{k}} U_{N}(x, t)=\frac{\partial^{k}}{\partial t^{k}} \mathcal{P}_{N} u_{e}(x, t)=\mathcal{P}_{N} \frac{\partial^{k} u_{e}(x, t)}{\partial t^{k}} .
$$

Consequently, the following linear estimates are straightforward:

$$
\begin{aligned}
& \left\|\partial_{t}\left(U_{N}-u_{e}\right)\right\|_{L^{2}} \leq C h^{m}\left\|\partial_{t} u_{e}\right\|_{H^{m}}, \\
& \left\|\partial_{x}\left(U_{N}-u_{e}\right)\right\|_{L^{2}} \leq C h^{m}\left\|u_{e}\right\|_{H^{m+1}}, \\
& \left\|\partial_{t} \partial_{x}^{2}\left(U_{N}-u_{e}\right)\right\|_{L^{2}} \leq C h^{m}\left\|\partial_{t} u_{e}\right\|_{H^{m+2}} .
\end{aligned}
$$

Again, a discrete $\|\cdot\|_{2}$ estimate should be given in the local truncation derivation. Note that

$$
\left\|\partial_{t}\left(U_{N}-u_{e}\right)\right\|_{2}=\left\|\mathcal{I}_{N}\left(\partial_{t}\left(U_{N}-u_{e}\right)\right)\right\|_{L^{2}} \leq\left\|\partial_{t}\left(U_{N}-u_{e}\right)\right\|_{L^{2}}+\left\|\partial_{t}\left(\mathcal{I}_{N} u_{e}-u_{e}\right)\right\|_{L^{2}},
$$

where the fact $\mathcal{I}_{N} \partial_{t} U_{N}=\partial_{t} U_{N}$ is applied, since $\partial_{t} U_{N} \in \mathcal{B}^{N}$. The first term on the righthand side of (3.8) has an estimate obtained from (3.5), and it follows from (2.2) that the second term also is bounded by

$$
\left\|\partial_{t}\left(\mathcal{I}_{N} u_{e}-u_{e}\right)\right\|_{L^{2}}=\left\|\mathcal{I}_{N} \partial_{t} u_{e}-\partial_{t} u_{e}\right\|_{L^{2}} \leq C h^{m}\left\|\partial_{t} u_{e}\right\|_{H^{m}}
$$

Therefore, from (3.5), (3.8), and (3.9), we have

$$
\left\|\partial_{t}\left(U_{N}-u_{e}\right)\right\|_{2} \leq C h^{m}\left\|\partial_{t} u_{e}\right\|_{H^{m}}
$$


Similarly, we have

$$
\left\|\partial_{x}\left(U_{N}-u_{e}\right)\right\|_{2} \leq C h^{m}\left\|u_{e}\right\|_{H^{m+1}}, \quad\left\|\partial_{t} \partial_{x}^{2}\left(U_{N}-u_{e}\right)\right\|_{2} \leq C h^{m}\left\|\partial_{t} u_{e}\right\|_{H^{m+2}}
$$

For the nonlinear term, from the expansion $\partial_{x} u_{e}^{p}=p u_{e}^{p-1}\left(u_{e}\right)_{x}$ and

$$
\begin{aligned}
\partial_{x}\left(u_{e}^{p}-U_{N}^{p}\right) & =p u_{e}^{p-1}\left(u_{e}\right)_{x}-p U_{N}^{p-1}\left(U_{N}\right)_{x} \\
& =p U_{N}^{p-1}\left(u_{e}-U_{N}\right)_{x}+p\left(u_{e}^{p-1}-U_{N}^{p-1}\right)\left(u_{e}\right)_{x} \\
& =p U_{N}^{p-1}\left(u_{e}-U_{N}\right)_{x}+p\left(u_{e}\right)_{x}\left(u_{e}-U_{N}\right) \sum_{k=0}^{p-2} u_{e}^{k} U_{N}^{p-2-k},
\end{aligned}
$$

we get

$$
\begin{aligned}
& \left\|\partial_{x}\left(u_{e}^{p}-U_{N}^{p}\right)\right\|_{L^{2}} \\
& \quad \leq C\left(\left\|U_{N}\right\|_{L^{\infty}}^{p-1}\left\|u_{e}-U_{N}\right\|_{H^{1}}+\left\|u_{e}-U_{N}\right\|_{L^{\infty}}\left(\left\|u_{e}\right\|_{L^{\infty}}^{p-2}+\left\|U_{N}\right\|_{L^{\infty}}^{p-2}\right)\left\|u_{e}\right\|_{H^{1}}\right) \\
& \quad \leq C\left\|u_{e}-U_{N}\right\|_{H^{1}}\left\|u_{e}\right\|_{H^{1}}^{p} \leq C h^{m}\left\|u_{e}\right\|_{H^{m+1}}\left\|u_{e}\right\|_{H^{1}}^{p},
\end{aligned}
$$

in which the 1D Sobolev embedding is used.

Furthermore, from (2.2), the following interpolation error also can be derived in a similar analysis:

$$
\begin{aligned}
& \left\|\partial_{x} u_{e}^{p}-\mathcal{I}_{N}\left(\partial_{x} u_{e}^{p}\right)\right\|_{L^{2}} \leq C h^{m}\left\|\partial_{x} u_{e}^{p}\right\|_{H^{m}} \leq C h^{m}\left\|u_{e}\right\|_{H^{1}}^{p}\left\|u_{e}\right\|_{H^{m+1}}, \\
& \left\|\partial_{x} U_{N}^{p}-\mathcal{I}_{N}\left(\partial_{x} U_{N}^{p}\right)\right\|_{L^{2}} \leq C h^{m}\left\|\partial_{x} U_{N}^{p}\right\|_{H^{m}} \leq C h^{m}\left\|u_{e}\right\|_{H^{1}}^{p}\left\|u_{e}\right\|_{H^{m+1}}
\end{aligned}
$$

Then the combination of (3.12), (3.13), and (3.14) yields

$$
\begin{aligned}
\left\|\partial_{x}\left(u_{e}^{p}-U_{N}^{p}\right)\right\|_{2} & =\left\|\mathcal{I}_{N} \partial_{x}\left(u_{e}^{p}-U_{N}^{p}\right)\right\|_{L^{2}} \\
& \left.=\| \partial_{x}\left(u_{e}^{p}-U_{N}^{p}\right)-\partial_{x} u_{e}^{p}+\mathcal{I}_{N} \partial_{x} u_{e}^{p}+\partial_{x} U_{N}^{p}-\mathcal{I}_{N} \partial_{x} U_{N}^{p}\right) \|_{L^{2}} \\
& \leq\left\|\partial_{x}\left(u_{e}^{p}-U_{N}^{p}\right)\right\|_{L^{2}}+\left\|\partial_{x} u_{e}^{p}-\mathcal{I}_{N}\left(\partial_{x} u_{e}^{p}\right)\right\|_{L^{2}}+\left\|\partial_{x} U_{N}^{p}-\mathcal{I}_{N}\left(\partial_{x} U_{N}^{p}\right)\right\|_{L^{2}} \\
& \leq C h^{m}\left\|u_{e}\right\|_{H^{1}}^{p}\left\|u_{e}\right\|_{H^{m+1}}
\end{aligned}
$$

Thus, the bounds on the projection error and its derivatives indicate that

$$
\partial_{t} U_{N}-\beta \partial_{x x t} U_{N}+\partial_{x} U_{N}+\alpha \partial_{x} U_{N}^{p}=\partial_{t} u_{e}-\beta \partial_{x x t} u_{e}+\partial_{x} u_{e}+\alpha \partial_{x} u_{e}^{p}+\tau_{1}=\tau_{1}
$$

with $\left\|\tau_{1}\right\|_{2} \leq C h^{m}$, since $u_{e}$ is the exact solution.

\subsection{Truncation error analysis in time}

For simplicity of presentation, we assume $T=K \Delta t$ with an integer $K$. Our approach is based upon the following simple, but fundamental estimates [33].

Lemma 3.1 [33] For $f \in H^{3}(0, T)$, we have

$$
\left\|\tau^{t} f\right\|_{l^{2}(0, T)} \leq C \Delta t^{m}\|f\|_{H^{m+1}(0, T)},
$$


with $\tau^{t} f^{n}=\frac{f^{n+1}-f^{n}}{\Delta t}-f^{\prime}\left(t^{n+1 / 2}\right)$, for $0 \leq m \leq 2$, where $C$ only depends on $T,\|\cdot\|_{l^{2}(0, T)}$ is a discrete $L^{2}$ norm (in time) given by $\|g\|_{l^{2}(0, T)}=\sqrt{\Delta t \sum_{n=0}^{K-1}\left(g^{n}\right)^{2}}$.

Lemma 3.2 [33] For $f \in H^{2}(0, T)$, we have

$$
\begin{aligned}
& \left\|D_{t / 2}^{2} f\right\|_{l^{2}(0, T)}:=\left(\Delta t \sum_{n=0}^{K-1}\left(D_{t / 2}^{2} f^{n+1 / 2}\right)^{2}\right)^{\frac{1}{2}} \leq C\|f\|_{H^{2}(0, T)}, \\
& \left\|D_{t}^{2} f\right\|_{l^{2}(0, T)}:=\left(\Delta t \sum_{n=0}^{K-1}\left(D_{t}^{2} f^{n}\right)^{2}\right)^{\frac{1}{2}} \leq C\|f\|_{H^{2}(0, T)}, \\
& \text { with } D_{t / 2}^{2} f^{n+1 / 2}=\frac{4\left(f^{n+1}-2 f\left(\cdot, t^{n+1 / 2}\right)+f^{n}\right)}{\Delta t^{2}}, \quad D_{t}^{2} f^{n}=\frac{f^{n+1}-2 f^{n}+f^{n-1}}{\Delta t^{2}},
\end{aligned}
$$

where $C$ only depends on $T$.

For the approximate solution $U_{N}$, define its vector grid function $U^{n}=\mathcal{I} U_{N}$ as its interpolation: $U_{i}^{n}=U_{N}^{n}\left(x_{i}, t^{n}\right)$.

Theorem 3.1 For any fixed time $T$, assume that the problem (1.1)-(1.3) possesses a unique solution $u_{e}$ such that $u_{e} \in L^{\infty}\left(0, T ; H^{m+1}\right) \cap H^{3}\left(0, T ; H^{2}\right)$. Let $U_{N}$ be the approximation solution and $U$ be its discrete interpolation. Then we have

$$
\begin{gathered}
\left(1-\beta \mathcal{D}_{N}^{2}\right) \frac{U^{n+1}-U^{n}}{\Delta t}+\mathcal{D}_{N}\left(\frac{3}{2} U^{n}-\frac{1}{2} U^{n-1}\right) \\
+\alpha \mathcal{D}_{N}\left(\left(\frac{3}{2}\left(U^{n}\right)^{p}-\frac{1}{2}\left(U^{n-1}\right)^{p}\right)\right)=\tau^{n},
\end{gathered}
$$

where $\tau^{k}$ satisfies

$$
\|\tau\|_{l^{2}\left(0, T ; l^{2}\right)}:=\left(\Delta t \sum_{k=0}^{K}\left\|\tau^{k}\right\|_{2}^{2}\right)^{1 / 2} \leq C\left(\Delta t^{2}+h^{m}\right),
$$

in which $C$ only depends on the regularity of the exact solution $u_{e}$.

Proof According to Lemma 3.1, letting $m=2$, we have

$$
\frac{U_{N}^{n+1}-U_{N}^{n}}{\Delta t}=\partial_{t} U_{N}\left(\cdot, t^{n+\frac{1}{2}}\right)+\tau_{2}^{n}(\cdot)
$$

with $\left\|\tau_{2}\right\|_{l^{2}(0, T)} \leq C \Delta t^{2}\left\|U_{N}(\cdot)\right\|_{H^{3}(0, T)} \leq C \Delta t^{2}\left\|u_{e}\right\|_{H^{3}(0, T)}$. In turn, an application of a discrete summation in $\Omega$ yields

$$
\left\|\tau_{2}\right\|_{l^{2}\left(0, T ; l^{2}\right)} \leq C \Delta t^{2}\left\|U_{N}(\cdot)\right\|_{H^{3}\left(0, T ; L^{2}\right)} \leq C \Delta t^{2}\left\|u_{e}\right\|_{H^{3}\left(0, T ; L^{2}\right)} .
$$

Using a similar argument, we also can arrive at

$$
\frac{\partial_{x}^{2} U_{N}^{n+1}-\partial_{x}^{2} U_{N}^{n}}{\Delta t}=\partial_{x x t} U_{N}\left(\cdot, t^{n+\frac{1}{2}}\right)+\tau_{3}^{n}(\cdot)
$$


which leads to

$$
\begin{aligned}
\left\|\tau_{3}\right\|_{l^{2}\left(0, T ; l^{2}\right)} & \leq C \Delta t^{2}\left\|\partial_{x}^{2} U_{N}(\cdot)\right\|_{H^{3}\left(0, T ; L^{2}\right)} \leq C \Delta t^{2}\left\|U_{N}(\cdot)\right\|_{H^{3}\left(0, T ; H^{2}\right)} \\
& \leq C \Delta t^{2}\left\|u_{e}\right\|_{H^{3}\left(0, T ; H^{2}\right)} .
\end{aligned}
$$

Since $\left(\frac{3}{2} U_{N}^{n}-\frac{1}{2} U_{N}^{n-1}\right) \in \mathcal{B}^{N}$, the following result is valid:

$$
\left\|\mathcal{D}_{N}\left(\frac{3}{2} U^{n}-\frac{1}{2} U^{n-1}\right)-\mathcal{I}\left(\partial_{x}\left(\frac{3}{2} U_{N}^{n}-\frac{1}{2} U_{N}^{n-1}\right)\right)\right\|_{2}=0,
$$

where $\mathcal{I}$ is the discrete operator of the continuous function at fixed points. Also, one can observe that

$$
\frac{3}{2} U_{N}^{n}-\frac{1}{2} U_{N}^{n-1}=U_{N}\left(\cdot, t^{n+\frac{1}{2}}\right)+\frac{1}{8} \Delta t^{2} D_{t / 2}^{2} U_{N}-\frac{1}{2} \Delta t^{2} D_{t}^{2} U_{N}
$$

and denote

$$
\partial_{x}\left(\frac{3}{2} U_{N}^{n}-\frac{1}{2} U_{N}^{n-1}\right)=\partial_{x} U_{N}\left(\cdot, t^{n+\frac{1}{2}}\right)+\tau_{4}^{n}(\cdot)
$$

An application of Lemma 3.2 also yields

$$
\left\|D_{t / 2}^{2} \partial_{x} U_{N}\right\|_{l^{2}(0, T)} \leq C\left\|\partial_{x} U_{N}\right\|_{H^{2}(0, T)}, \quad\left\|D_{t}^{2} \partial_{x} U_{N}\right\|_{l^{2}(0, T)} \leq C\left\|\partial_{x} U_{N}\right\|_{H^{2}(0, T)}
$$

As a result, the combination of (3.26)-(3.29) implies that

$$
\left\|\tau_{4}\right\|_{l^{2}\left(0, T ; l^{2}\right)} \leq C \Delta t^{2}\left\|U_{N}(\cdot)\right\|_{H^{2}\left(0, T ; H^{1}\right)} \leq C \Delta t^{2}\left\|u_{e}\right\|_{H^{2}\left(0, T ; H^{1}\right)} .
$$

For the nonlinear term, let

$$
\partial_{x}\left(\frac{3}{2}\left(U_{N}^{p}\right)^{n}-\frac{1}{2}\left(U_{N}^{p}\right)^{n-1}\right)=\partial_{x} U_{N}^{p}\left(\cdot, t^{n+\frac{1}{2}}\right)+\tau_{5}^{n}(\cdot) .
$$

First, we have the following estimate:

$$
\begin{aligned}
\| \mathcal{D}_{N} & \left(\frac{3}{2}\left(U^{p}\right)^{n}-\frac{1}{2}\left(U^{p}\right)^{n-1}\right)-\mathcal{I}\left(\partial_{x}\left(\frac{3}{2}\left(U_{N}^{p}\right)^{n}-\frac{1}{2}\left(U_{N}^{p}\right)^{n-1}\right)\right) \|_{2} \\
& \leq C h^{m}\left\|\frac{3}{2}\left(U_{N}^{p}\right)^{n}-\frac{1}{2}\left(U_{N}^{p}\right)^{n-1}\right\|_{H^{m+1}} \\
& \leq C h^{m}\left(\left\|U_{N}^{n}\right\|_{H^{m+1}}^{p}+\left\|U_{N}^{n-1}\right\|_{H^{m+1}}^{p}\right) \\
& \leq C h^{m}\left\|U_{N}\right\|_{L^{\infty}\left(0, T ; H^{m+1}\right)^{p}}^{p}
\end{aligned}
$$

Similar to (3.27), one can get

$$
\partial_{x}\left(\frac{3}{2}\left(U_{N}^{p}\right)^{n}-\frac{1}{2}\left(U_{N}^{p}\right)^{n-1}\right)=\partial_{x} U_{N}^{p}\left(\cdot, t^{n+\frac{1}{2}}\right)+\frac{1}{8} \Delta t^{2} D_{t / 2}^{2} \partial_{x} U_{N}^{p}-\frac{1}{2} \Delta t^{2} D_{t}^{2} \partial_{x} U_{N}^{p},
$$


which leads to

$$
\begin{aligned}
& \left\|\mathcal{I}\left(\partial_{x}\left(\frac{3}{2}\left(U_{N}^{p}\right)^{n}-\frac{1}{2}\left(U_{N}^{p}\right)^{n-1}\right)-\partial_{x} U_{N}^{p}\left(\cdot, t^{n+\frac{1}{2}}\right)\right)\right\|_{2} \\
& \quad \leq \frac{1}{8} \Delta t^{2}\left\|D_{t / 2}^{2} \partial_{x} U_{N}^{p}\right\|_{H^{2}}+\frac{1}{2} \Delta t^{2}\left\|D_{t}^{2} \partial_{x} U_{N}^{p}\right\|_{H^{2}},
\end{aligned}
$$

where the estimate (2.2) is applied in the last step. Meanwhile, using the Hölder inequality, the Sobolev imbedding, and the first- and the second-order time derivative of $U_{N}^{p}$, we can get

$$
\left\|U_{N}^{p}\right\|_{H^{2}(0, T)} \leq C\left\|U_{N}\right\|_{H^{2}(0, T)}^{p} .
$$

With the same analysis above of the term $\left\|\tau_{4}\right\|_{2}$, the direct estimate of $\left\|\tau_{5}\right\|_{2}$ is given as follows:

$$
\left\|\tau_{5}\right\|_{l^{2}\left(0, T ; l^{2}\right)} \leq C \Delta t^{2}\left\|U_{N}(\cdot)\right\|_{H^{2}\left(0, T ; H^{1}\right)} \leq C \Delta t^{2}\left\|u_{e}\right\|_{H^{2}\left(0, T ; H^{1}\right)} .
$$

Therefore, the second order in time consistency of the approximation solution $U$ is obtained. With the truncation error analysis of $U_{N}$, we can get the result (3.21), in which $\tau=\mathcal{I}_{N}\left(\tau_{1}+\tau_{2}+\beta \tau_{3}+\tau_{4}+\alpha \tau_{5}\right)$. This completes the detailed consistency analysis.

\section{Stability and convergence}

Let $e_{i}^{n}=U_{i}^{n}-u_{i}^{n}$, and denote by $u_{N}^{n} \in B^{N}, e_{N}^{n} \in B^{N}$ the continuous versions of the numerical solution $u^{n}$ and $e^{n}$, with the interpolation formula given by (2.1), respectively.

The following lemma is critical to our analysis and enables us to obtain an $H^{m}$ bound of the interpolation of the nonlinear term (for detailed proof, see [29]).

Lemma 4.1 [29] For any $f \in \mathcal{B}^{p N}$ (with $p$ an integer) in dimension $d$, we have

$$
\left\|\mathcal{I}_{N} f\right\|_{H^{k}} \leq(\sqrt{p})^{d}\|f\|_{H^{k}}
$$

Moreover, the following preliminary estimate also will be used in the later analysis.

Lemma 4.2 At any time step $t^{k}, k \geq 0$, we have

$$
\left\|e_{N}^{k}\right\|_{H^{1}} \leq C_{1}\left(\left\|\mathcal{D}_{N} e^{k}\right\|_{2}+h^{m}\right)
$$

Proof From the Poincaré inequality, we have

$$
\left\|e_{N}^{k}\right\|_{H^{1}} \leq C_{1}\left(\left\|\partial_{x} e_{N}^{k}\right\|_{L^{2}}+\left|\int_{\Omega} e_{N}^{k} d x\right|\right)
$$

Since $U_{N}^{k}, u_{N}^{k} \in \mathcal{B}^{N}, e_{N}^{k}=U_{N}^{k}-u_{N}^{k} \in \mathcal{B}^{N}$ holds, which also shows that

$$
\left\|\partial_{x} e_{N}^{k}\right\|_{L^{2}}=\left\|\mathcal{D}_{N} e^{k}\right\|_{2}
$$


On the other hand, we recall that the solution of the GRLW equation (1.1) is mass conservative:

$$
\int_{\Omega} u_{e}(\cdot, t) d x=\int_{\Omega} u_{e}(\cdot, 0) d x
$$

for $\forall t>0$. Considering $U_{N}$ is the projection of $u_{e}$ into $\mathcal{B}^{N}$, we have

$$
\int_{\Omega} U_{N}(\cdot, t) d x=\int_{\Omega} u_{e}(\cdot, t) d x=\int_{\Omega} u_{e}(\cdot, 0) d x=\int_{\Omega} U_{N}(\cdot, 0) d x
$$

for $\forall t>0$. Conversely, the numerical scheme (2.6) is mass conservative: $\overline{u^{k}}:=h \sum_{i=0}^{N-1} u_{i}^{k}=$ $\overline{u^{0}}$. For $U_{N}^{k} \in \mathcal{B}^{N}$, one can get

$$
\overline{U^{k}}=\int_{\Omega} U_{N}\left(\cdot, t^{k}\right) d x=\int_{\Omega} U_{N}(\cdot, 0) d x=\overline{U^{0}}
$$

Owing to the spectral accuracy in space for the numerical error function at each time step, we obtain

$$
\overline{e^{k}}=\overline{U^{k}-u^{k}}=\overline{U^{k}}-\overline{u^{k}}=\overline{U^{0}}-\overline{u^{0}}=O\left(h^{m}\right), \quad \forall k>0
$$

which is equivalent to

$$
\int_{\Omega} e_{N}^{k} d x=\overline{e^{k}}=O\left(h^{m}\right)
$$

Hence, substituting (4.4) and (4.5) into (4.3) yields the inequality (4.2).

Suppose the constructed approximation solution has a $W^{1, \infty}$ bound,

$$
\left\|U_{N}\right\|_{L^{\infty}\left(0, T ; W^{1, \infty}\right)}=\sup _{0 \leq t \leq T}\left(\left\|u_{N}\right\|_{L^{\infty}}+\left\|\left(u_{N}\right)_{x}\right\|_{L^{\infty}}\right) \leq C_{2},
$$

i.e.,

$$
\left\|U_{N}^{n}\right\|_{L^{\infty}} \leq C_{2}, \quad\left\|\left(U_{N}\right)_{x}^{n}\right\|_{L^{\infty}} \leq C_{2},
$$

for any $n \geq 0$, which comes from the regularity of the constructed solution.

Now, we present the main result of this paper.

Theorem 4.1 For any fixed time $T$, assume that the exact solution $u_{e}$ for the GRLW equations (1.1)-(1.3) satisfies $u_{e} \in L^{\infty}\left(0, T ; H^{m+1}\right) \cap H^{3}\left(0, T ; H^{2}\right)$. Denote by $u_{\Delta t, h}$ the continuous extension of the fully discrete numerical solution computed by the proposed scheme (2.6). Then, as $\Delta t, h \rightarrow 0$, we have the following convergence result:

$$
\left\|u_{\Delta t, h}-u_{e}\right\|_{l^{\infty}\left(0, T ; H^{1}\right)} \leq \tilde{C}\left(\Delta t^{2}+h^{m}\right)
$$

provided that the time step $\Delta t$ and the space grid size $h$ are bounded by given constants. Note that the convergence constant $\tilde{C}$ also depends on the exact solution and the fixed time $T$. 
Proof We have an a priori $H^{1}$ assumption up to time step $t^{n}$.

Assume that the numerical error function has an $H^{1}$ bound at time steps $t^{n}, t^{n-1}$,

$$
\left\|e_{N}^{k}\right\|_{H^{1}} \leq 1, \quad \text { with } e_{N}^{k}=\mathcal{I}_{N} e^{k}, \text { for } k=n, n-1,
$$

which yields directly the following results:

$$
\begin{aligned}
& \left\|u_{N}^{k}\right\|_{H^{1}}=\left\|U_{N}^{k}-e_{N}^{k}\right\|_{H^{1}} \leq C_{2}+1:=C_{3}, \\
& \left\|u^{k}\right\|_{\infty} \leq C\left\|u_{N}^{k}\right\|_{L^{\infty}} \leq C\left\|u_{N}^{k}\right\|_{H^{1}} \leq C C_{3}:=C_{4},
\end{aligned}
$$

where a 1-D Sobolev embedding was used in the last step.

Subtracting (2.6) from (3.20), we have

$$
\begin{aligned}
\left(1-\beta \mathcal{D}_{N}^{2}\right) \frac{e^{n+1}-e^{n}}{\Delta t}= & -\mathcal{D}_{N}\left(\frac{3}{2} e^{n}-\frac{1}{2} e^{n-1}\right)-\alpha \mathcal{D}_{N}\left(\frac{3}{2} e^{n} \sum_{k=0}^{p-1}\left(U^{n}\right)^{k}\left(u^{n}\right)^{p-1-k}\right. \\
& \left.-\frac{1}{2} e^{n-1} \sum_{k=0}^{p-1}\left(U^{n-1}\right)^{k}\left(u^{n-1}\right)^{p-1-k}\right)+\tau^{n} .
\end{aligned}
$$

For convenience, denote

$$
\begin{aligned}
\mathcal{N} \mathcal{L} \mathcal{T}_{1} & =-\frac{3}{2} e^{n} \sum_{k=0}^{p-1}\left(U^{n}\right)^{k}\left(u^{n}\right)^{p-1-k}, \\
\mathcal{N} \mathcal{L} \mathcal{T}_{2} & =\frac{1}{2} e^{n-1} \sum_{k=0}^{p-1}\left(U^{n-1}\right)^{k}\left(u^{n-1}\right)^{p-1-k} .
\end{aligned}
$$

Taking a discrete $L^{2}$ inner product of (4.10) with $2 e^{n+1}$, we get

$$
\begin{aligned}
\langle(1- & \left.\left.\beta \mathcal{D}_{N}^{2}\right)\left(e^{n+1}-e^{n}\right), 2 e^{n+1}\right\rangle \\
= & -\Delta t\left\langle\mathcal{D}_{N}\left(\frac{3}{2} e^{n}-\frac{1}{2} e^{n-1}\right), 2 e^{n+1}\right\rangle \\
& +\Delta t \alpha\left(\left\langle\mathcal{D}_{N}\left(\mathcal{N} \mathcal{L} \mathcal{T}_{1}\right), 2 e^{n+1}\right\rangle+\left\langle\mathcal{D}_{N}\left(\mathcal{N} \mathcal{L}_{2}\right), 2 e^{n+1}\right\rangle\right)+\Delta t\left\langle\tau^{n}, 2 e^{n+1}\right\rangle .
\end{aligned}
$$

The first term on the left side of (4.13) and the truncation error term can be handled in a straightforward way as follows:

$$
\begin{aligned}
& \left\langle e^{n+1}-e^{n}, 2 e^{n+1}\right\rangle=\left\|e^{n+1}\right\|_{2}^{2}-\left\|e^{n}\right\|_{2}^{2}+\left\|e^{n+1}-e^{n}\right\|_{2}^{2}, \\
& -\beta\left\langle\mathcal{D}_{N}^{2}\left(e^{n+1}-e^{n}\right), 2 e^{n+1}\right\rangle=2 \beta\left\langle\mathcal{D}_{N}\left(e^{n+1}-e^{n}\right), \mathcal{D}_{N} e^{n+1}\right\rangle, \\
& =\beta\left\|\mathcal{D}_{N} e^{n+1}\right\|_{2}^{2}-\beta\left\|\mathcal{D}_{N} e^{n}\right\|_{2}^{2}+\beta\left\|\mathcal{D}_{N} e^{n+1}-\mathcal{D}_{N} e^{n}\right\|_{2}^{2}, \\
& \left\langle\tau^{n}, 2 e^{n+1}\right\rangle \leq\left\|\tau^{n}\right\|_{2}^{2}+\left\|e^{n+1}\right\|_{2}^{2},
\end{aligned}
$$


in which a discrete Cauchy inequality is used in the last inequality. Similarly, for the first term on the right side of (4.13), one can get

$$
\begin{aligned}
-\left\langle\mathcal{D}_{N}\left(\frac{3}{2} e^{n}-\frac{1}{2} e^{n-1}\right), 2 e^{n+1}\right\rangle & =-3\left\langle\mathcal{D}_{N} e^{n}, e^{n+1}\right\rangle+\left\langle\mathcal{D}_{N} e^{n-1}, e^{n+1}\right\rangle \\
& \leq \frac{3}{2}\left\|\mathcal{D}_{N} e^{n}\right\|_{2}^{2}+\frac{1}{2}\left\|\mathcal{D}_{N} e^{n-1}\right\|_{2}^{2}+2\left\|e^{n+1}\right\|_{2}^{2} .
\end{aligned}
$$

Next, we discuss nonlinear terms on the right-hand side of (4.13) in turn.

For the nonlinear term $\mathcal{N} \mathcal{L} \mathcal{T}_{1}$, noting that

$$
e^{n}\left(U^{n}\right)^{k}\left(u^{n}\right)^{p-1-k}=\mathcal{I}\left(e_{N}^{n}\left(U_{N}^{n}\right)^{k}\left(u_{N}^{n}\right)^{p-1-k}\right), \quad 0 \leq k \leq p-1,
$$

we have

$$
\left\|\mathcal{D}_{N} e^{n}\left(U^{n}\right)^{k}\left(u^{n}\right)^{p-1-k}\right\|_{2}=\left\|\partial_{x}\left(\mathcal{I}_{N}\left(e_{N}^{n}\left(U_{N}^{n}\right)^{k}\left(u_{N}^{n}\right)^{p-1-k}\right)\right)\right\|_{L^{2}}, \quad 0 \leq k \leq p-1 .
$$

Since $e_{N}^{n}\left(U_{N}^{n}\right)^{k}\left(u_{N}^{n}\right)^{p-1-k} \in \mathcal{B}^{p N}$, an application of Lemma 4.1 yields

$$
\begin{aligned}
\left\|\partial_{x}\left(\mathcal{I}_{N}\left(e_{N}^{n}\left(U_{N}^{n}\right)^{k}\left(u_{N}^{n}\right)^{p-1-k}\right)\right)\right\|_{L^{2}} & \leq\left\|\mathcal{I}_{N}\left(e_{N}^{n}\left(U_{N}^{n}\right)^{k}\left(u_{N}^{n}\right)^{p-1-k}\right)\right\|_{H^{1}} \\
& \leq \sqrt{p}\left\|e_{N}^{n}\left(U_{N}^{n}\right)^{k}\left(u_{N}^{n}\right)^{p-1-k}\right\|_{H^{1}}
\end{aligned}
$$

Moreover, a detailed expansion for $\partial_{x} e_{N}^{n}\left(U_{N}^{n}\right)^{k}\left(u_{N}^{n}\right)^{p-1-k}$ indicates that

$$
\left\|\partial_{x}\left(e_{N}^{n}\left(U_{N}^{n}\right)^{k}\left(u_{N}^{n}\right)^{p-1-k}\right)\right\|_{L^{2}} \leq C_{5}\left(\left\|U_{N}^{n}\right\|_{H^{1}}^{p-1}+\left\|u_{N}^{n}\right\|_{H^{1}}^{p-1}+1\right)\left\|e_{N}^{n}\right\|_{H^{1}},
$$

which can be obtained by repeated application of the Sobolev embedding, the Hölder inequality, and the Young inequality. Substituting the bound (4.6) for the constructed solution $U_{N}$ and the a priori assumption (4.9) into (4.21), respectively, we get

$$
\left\|\left(e_{N}^{n}\left(U_{N}^{n}\right)^{k}\left(u_{N}^{n}\right)^{p-1-k}\right)\right\|_{H^{1}} \leq C_{5}\left(C_{2}^{p-1}+C_{3}^{p-1}+1\right)\left\|e_{N}^{n}\right\|_{H^{1}} .
$$

Consequently, it follows from (4.19), (4.20), and (4.22) that

$$
\left\|\mathcal{D}_{N}\left(e^{n}\left(U^{n}\right)^{k}\left(u^{n}\right)^{p-1-k}\right)\right\|_{2} \leq C_{5}\left(C_{2}^{p-1}+C_{3}^{p-1}+1\right) \sqrt{p}\left\|e_{N}^{n}\right\|_{H^{1}},
$$

for any $0 \leq k \leq p-1$, that is,

$$
\left\|\mathcal{D}_{N}\left(\mathcal{N} \mathcal{L} \mathcal{T}_{1}\right)\right\|_{2} \leq C_{6}\left\|e_{N}^{n}\right\|_{H^{1}}
$$

with $C_{6}=C_{5}\left(C_{2}^{p-1}+C_{3}^{p-1}+1\right) \sqrt{p}$.

Obviously, a similar analysis can be applied to $\mathcal{N} \mathcal{L} \mathcal{T}_{2}$ and we have

$$
\left\|\mathcal{D}_{N}\left(\mathcal{N} \mathcal{L} \mathcal{T}_{2}\right)\right\|_{2} \leq C_{6}\left\|e_{N}^{n-1}\right\|_{H^{1}}
$$

From (4.24) and (4.25), one can get

$$
\left\|\mathcal{D}_{N}(\mathcal{N} \mathcal{L} \mathcal{T})\right\|_{2}=\left\|\mathcal{D}_{N}\left(\mathcal{N} \mathcal{L} \mathcal{T}_{1}\right)\right\|_{2}+\left\|\mathcal{D}_{N}\left(\mathcal{N} \mathcal{L} \mathcal{T}_{2}\right)\right\|_{2} \leq C_{6}\left(\left\|e_{N}^{n}\right\|_{H^{1}}+\left\|e_{N}^{n-1}\right\|_{H^{1}}\right)
$$


From the result above, the nonlinear inner product can be analyzed as

$$
\begin{aligned}
\alpha\left\langle\mathcal{D}_{N}(\mathcal{N} \mathcal{L} \mathcal{T}), 2 e^{n+1}\right\rangle \leq & 2 \alpha\left\|\mathcal{D}_{N}(\mathcal{N} \mathcal{L} \mathcal{T})\right\|_{2}\left\|e^{n+1}\right\|_{2} \\
\leq & 2 \alpha C_{6}\left(\left\|e_{N}^{n}\right\|_{H^{1}}+\left\|e_{N}^{n-1}\right\|_{H^{1}}\right)\left\|e^{n+1}\right\|_{2} \\
\leq & \alpha C_{6}\left(\left\|e_{N}^{n}\right\|_{H^{1}}^{2}+\left\|e_{N}^{n-1}\right\|_{H^{1}}^{2}+2\left\|e^{n+1}\right\|_{2}^{2}\right) \\
\leq & \alpha C_{6} C_{1}^{2}\left\|\mathcal{D}_{N} e^{n}\right\|_{2}^{2}+\alpha C_{6} C_{1}^{2}\left\|\mathcal{D}_{N} e^{n-1}\right\|_{2}^{2}+2 \alpha C_{6} C_{1}^{2}\left\|e^{n+1}\right\|_{2}^{2} \\
& +2 \alpha C_{6} C_{1}^{2} h^{2 m} \\
= & C_{7}\left\|\mathcal{D}_{N} e^{n}\right\|_{2}^{2}+C_{7}\left\|\mathcal{D}_{N} e^{n-1}\right\|_{2}^{2}+2 C_{7}\left\|e^{n+1}\right\|_{2}^{2}+2 C_{7} h^{2 m}
\end{aligned}
$$

in which $C_{7}=\alpha C_{6} C_{1}^{2}$ and Lemma 4.2 are applied.

Substituting (4.14), (4.15), (4.16), (4.17), (4.27) into (4.13) indicates that

$$
\begin{aligned}
& \left\|e^{n+1}\right\|_{2}^{2}-\left\|e^{n}\right\|_{2}^{2}+\left\|e^{n+1}-e^{n}\right\|_{2}^{2}+\beta\left\|\mathcal{D}_{N} e^{n+1}\right\|_{2}^{2}-\beta\left\|\mathcal{D}_{N} e^{n}\right\|_{2}^{2}+\beta\left\|\mathcal{D}_{N} e^{n+1}-\mathcal{D}_{N} e^{n}\right\|_{2}^{2} \\
& \leq\left(3+2 C_{7}\right) \Delta t\left\|e^{n+1}\right\|_{2}^{2}+\left(\frac{3}{2}+C_{7}\right) \Delta t\left\|\mathcal{D}_{N} e^{n}\right\|_{2}^{2}+\left(\frac{1}{2}+C_{7}\right) \Delta t\left\|\mathcal{D}_{N} e^{n-1}\right\|_{2}^{2} \\
& \quad+2 C_{7} \Delta t h^{2 m}+\Delta t\left\|\tau^{n}\right\|_{2}^{2} .
\end{aligned}
$$

Summing in time gives

$$
\begin{aligned}
\left\|e^{n+1}\right\|_{2}^{2}+\sum_{k=0}^{n}\left\|e^{k+1}-e^{k}\right\|_{2}^{2}+\beta\left\|\mathcal{D}_{N} e^{n+1}\right\|_{2}^{2}+\beta \sum_{k=0}^{n}\left\|\mathcal{D}_{N} e^{k+1}-\mathcal{D} e^{k}\right\|_{2}^{2} \\
\leq\left(3+2 C_{7}\right) \Delta t \sum_{k=0}^{n}\left\|e^{k+1}\right\|_{2}^{2}+\left(2+2 C_{7}\right) \Delta t \sum_{k=0}^{n}\left\|\mathcal{D}_{N} e^{k}\right\|_{2}^{2}+\left(\frac{1}{2}+C_{7}\right) \Delta t\left\|\mathcal{D}_{N} e^{0}\right\|_{2}^{2} \\
\quad+\left\|e^{0}\right\|_{2}^{2}+\beta\left\|\mathcal{D}_{N} e^{0}\right\|_{2}^{2}+2 C_{7} \Delta t h^{2 m}+\Delta t\left\|\tau^{n}\right\|_{2}^{2} \\
\leq C_{8} \Delta t\left(\sum_{k=0}^{n}\left\|e^{k+1}\right\|_{2}^{2}+\beta \Delta t \sum_{k=0}^{n}\left\|\mathcal{D}_{N} e^{k}\right\|_{2}^{2}\right)+2 C_{7} \Delta t h^{2 m}+\Delta t\left\|\tau^{n}\right\|_{2}^{2}+C h^{2 m} .
\end{aligned}
$$

Here $C_{8}=\left(3+2 C_{7}\right)$, and the choice of $C_{7}$ satisfies with $\left(2+2 C_{7}\right) /\left(3+2 C_{7}\right) \leq \beta$. Note that we use the fact $\left\|e^{0}\right\|_{H_{h}^{1}} \leq h^{m}$, due to the collocation spectral approximation of the initial data. As a result, an application of discrete Gronwall inequality leads to the following convergence result:

$$
\left\|e^{l}\right\|_{2}^{2}+\beta\left\|\mathcal{D}_{N} e^{l}\right\|_{2} \leq \tilde{C}\left(\Delta t^{2}+h^{m}\right), \quad \forall 0 \leq l \leq K
$$

where $\tilde{C}=C e^{\frac{1}{2} C_{8} T}$.

\section{Recovery of the $H^{1}$ a priori bound:}

With the help of $l^{\infty}\left(0, T ; H^{1}\right)$ error estimate (4.30), we can see the a priori estimate (4.8) is also valid for the numerical error function $e_{N}$ at time step $t^{n+1}$, provided that

$$
\Delta t \leq(\tilde{C})^{-1 / 2}, \quad h \leq(\tilde{C})^{-1 / m} .
$$

Finally, a combination of (4.31) and the classical projection (3.2) yields (4.8). The proof of Theorem 4.1 is completed. 
Remark 4.1 One well-known challenge in the nonlinear analysis of pseudospectral scheme comes from the aliasing error. This work avoids an application of the inverse inequality but adopts an novel aliasing error control estimate given by Lemma 4.1. So we could obtain the estimate for its discrete $H^{1}$ norm.

\section{Numerical experiments}

The single solitary-wave solution of (1.1) is

$$
u(x, t)=A \operatorname{sech}^{\frac{2}{p-1}}\left[K\left(x-c t+x_{0}\right)\right]
$$

where

$$
A=\left[\frac{(p+1)(c-1)}{2 \alpha}\right]^{\frac{1}{p-1}}, \quad K=\frac{p-1}{2 \beta} \sqrt{\frac{c-1}{c}},
$$

and $c, x_{0}$ are arbitrary constants and $p \geq 2$ is an integer. The corresponding initial function can be rewritten

$$
u_{0}(x)=A \operatorname{sech}^{\frac{2}{p-1}}\left[K\left(x+x_{0}\right)\right]
$$

\subsection{Propagation of single solitary wave}

Here we take $x_{0}=0, \alpha=\frac{1}{2}, \beta=1, x_{L}=-30, x_{R}=30$, and $c=1.5$ in (5.1) and (5.2). Choose $N=128$ and $\Delta t=0.01$ to investigate the dynamics of a single solitary wave. The profile of a single solitary waves for the GRLW equation with $p=2$ and $p=8$ from time $t=0$ to 6 are depicted in Figure 1. It can be seen that the solitary wave moves to the right unchanged in which the solitary wave propagates in a stable fashion. Moreover, while $p$ increases, the speed velocity of the wave decreases and the amplitude increases, as time increases.

\subsection{Accuracy tests}

\subsubsection{Spectral accuracy in space}

To investigate the accuracy in space, we take $\Delta t=10^{-3}$ so that the temporal numerical error is negligible. $x_{L}, x_{R}, \alpha, \beta$, and $c$ are defined as in Section 5.1. With grid sizes from
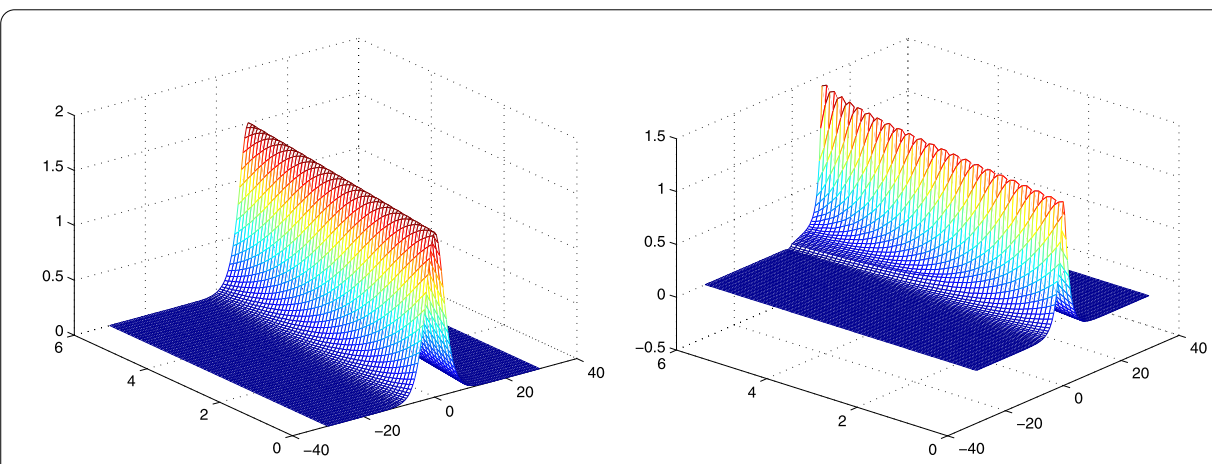

Figure 1 Profile of a single solitary wave $u(x, t)$ from $t=0$ to 6 with $p=2, c=1.5$ and $p=8, c=1.5$, respectively. 
Figure 2 Discrete $L^{2}$ numerical errors of $u$ with $\Delta t=10^{-3}$ for different $p$.

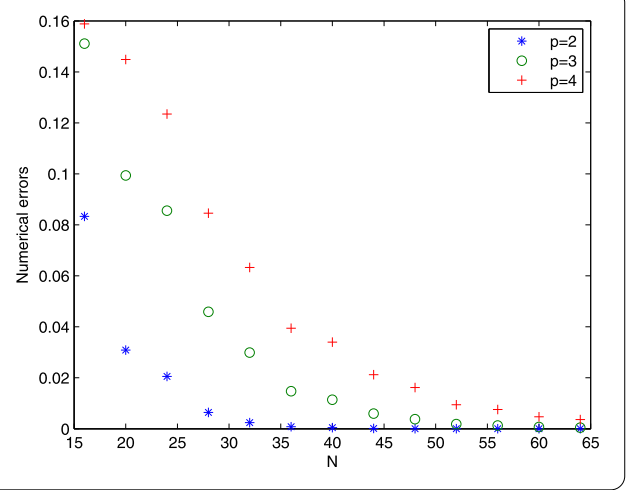

Table $1\|\cdot\|_{2}$ errors and convergence orders of numerical solution $u$ at $T=5$ for $p=2,4$, and 6

\begin{tabular}{lllllll}
\hline $\boldsymbol{\Delta t}$ & $\boldsymbol{p = 2}$ & order & $\boldsymbol{p = 4}$ & order & $\boldsymbol{p}=\mathbf{6}$ & order \\
\hline 0.1 & $2.2055199 \mathrm{e}-3$ & - & $7.4971272 \mathrm{e}-3$ & - & $2.4952949 \mathrm{e}-2$ & - \\
0.05 & $5.5175158 \mathrm{e}-4$ & 1.9990 & $1.6977751 \mathrm{e}-3$ & 2.1426 & $4.2548344 \mathrm{e}-3$ & 2.5520 \\
0.025 & $1.3823443 \mathrm{e}-4$ & 1.9969 & $4.0822074 \mathrm{e}-4$ & 2.0562 & $9.1684708 \mathrm{e}-4$ & 2.2143 \\
0.0125 & $3.4646085 \mathrm{e}-5$ & 1.9963 & $1.0027214 \mathrm{e}-4$ & 2.0254 & $2.1345592 \mathrm{e}-4$ & 2.1027 \\
0.00625 & $8.8159403 \mathrm{e}-6$ & 1.9745 & $2.4863890 \mathrm{e}-5$ & 2.0117 & $5.1499083 \mathrm{e}-5$ & 2.0513 \\
\hline
\end{tabular}

$N=16$ to 64 in increment of 4 , we solve (2.6) up to time $T=5$ for $p=2,3,4$, respectively. The discrete norm $\|\cdot\|_{2}$ of numerical errors at $T=5$ is given in Figure 2, which shows apparently the spatial spectral accuracy is verified.

\subsubsection{Second-order accuracy in time}

For exploring the temporal accuracy, we fix spatial resolution as $N=1,024$ so that the numerical error is dominated mainly by the temporal ones. With a sequence of time step sizes $\Delta t=0.1,0.05,0.025,0.0125,0.00625$, we also compute the numerical errors at $T=5$ for $p=2,4,6$, respectively, and the results are presented in Table 1 in which a second-order in time accuracy is shown clearly.

\subsubsection{Conservation properties}

As stated in [34, 35], the GRLW equation has three conservative laws related to mass, momentum and energy, respectively. In particular, for $p=2$, it reduces to the regularized long wave (RLW) equation. The invariants are given by

$$
I_{1}=\int_{\Omega} u d x, \quad I_{2}=\int_{\Omega}\left(u^{2}+\beta u_{x}^{2}\right) d x, \quad I_{3}=\int_{\Omega}\left(u^{3}+3 u^{2}\right) d x,
$$

and the corresponding discrete quantities are computed for the numerical scheme as follows:

$$
\begin{aligned}
& \mathcal{I}_{1}=h \sum_{j=0}^{2 N} u_{j}^{n}, \quad \mathcal{I}_{2}=h \sum_{j=0}^{2 N}\left[\left(u_{j}^{n}\right)^{2}+\beta\left(\left(u_{x}\right)_{j}^{n}\right)^{2}\right], \\
& \mathcal{I}_{3}=h \sum_{j=0}^{2 N}\left[\left(u_{j}^{n}\right)^{3}+3\left(u_{j}^{n}\right)^{2}\right] .
\end{aligned}
$$


Table 2 Invariants for single soliton of RLW equation with $N=128$ and $c=\frac{4}{3}$

\begin{tabular}{|c|c|c|c|c|c|c|}
\hline \multirow[t]{2}{*}{ Time } & \multicolumn{3}{|l|}{$\Delta t=0.05$} & \multicolumn{3}{|l|}{$\Delta t=0.025$} \\
\hline & $\overline{\mathcal{I}_{1}}$ & $\mathcal{I}_{2}$ & $\mathcal{I}_{3}$ & $\overline{\mathcal{I}_{1}}$ & $\mathcal{I}_{2}$ & $\mathcal{I}_{3}$ \\
\hline$T=0$ & 7.9999950 & 5.5997821 & 20.266666 & 7.9999950 & 5.5997821 & 20.266666 \\
\hline$T=4$ & 7.9999950 & 5.5998333 & 20.266871 & 7.9999950 & 5.5997884 & 20.266692 \\
\hline$T=$ & 7.9999950 & 5.5998850 & 20.267078 & 7.9999950 & 5.5997947 & 20.266717 \\
\hline$T=12$ & 7.9999950 & 5.5999359 & 20.267284 & 7.9999950 & 5.5998002 & 20.266743 \\
\hline$T=16$ & 7.9999950 & 5.5998258 & 20.267490 & 7.9999950 & 5.5996448 & 20.266768 \\
\hline
\end{tabular}

Table 3 Invariants for single soliton of MRLW equation with $N=128$ and $c=\frac{4}{3}$

\begin{tabular}{|c|c|c|c|c|c|c|}
\hline \multirow[t]{2}{*}{ Time } & \multicolumn{3}{|l|}{$\Delta t=0.05$} & \multicolumn{3}{|l|}{$\Delta t=0.025$} \\
\hline & $\mathcal{J}_{1}$ & $\mathcal{J}_{2}$ & $\mathcal{J}_{3}$ & $\overline{\mathcal{J}_{1}}$ & $\mathcal{J}_{2}$ & $\mathcal{J}_{3}$ \\
\hline$T=0$ & 7.9999950 & 5.5997821 & 2.0584499 & 7.9999950 & 5.5997821 & 2.0584499 \\
\hline$T=4$ & 7.9999950 & 5.5998333 & 2.0586695 & 7.9999950 & 5.5997884 & 2.0584982 \\
\hline$T=8$ & 7.9999950 & 5.5998850 & 2.0588246 & 7.9999950 & 5.5997947 & 2.0585311 \\
\hline$T=12$ & 7.9999950 & 5.5999359 & 2.05886249 & 7.9999950 & 5.5998002 & 2.0585384 \\
\hline$T=16$ & 7.9999950 & 5.5998258 & 2.0598310 & 7.9999950 & 5.5996448 & 2.0595023 \\
\hline
\end{tabular}

Take $\Delta t=0.05$ and $\Delta t=0.025$ with $N=128$ and $c=\frac{4}{3} \cdot x_{L}, x_{R}, \alpha, \beta$ are also defined as in Section 5.1. The invariants $\mathcal{I}_{1}, \mathcal{I}_{2}$, and $\mathcal{I}_{3}$ up to time $T=16$ are given in Table 2.

Another special case of the GRLW equation is the modified regularized long wave (MRLW) equation with $p=3$. Similar to the quantities for the RLW equation, the MRLW equation also has the following conservative laws:

$$
J_{1}=\int_{\Omega} u d x, \quad J_{2}=\int_{\Omega}\left(u^{2}+\beta u_{x}^{2}\right) d x, \quad J_{3}=\int_{\Omega}\left(u^{4}-6 \beta u_{x}^{2}\right) d x
$$

and

$$
\begin{aligned}
& \mathcal{J}_{1}=h \sum_{j=0}^{2 N} u_{j}^{n}, \quad \mathcal{J}_{2}=h \sum_{j=0}^{2 N}\left[\left(u_{j}^{n}\right)^{2}+\beta\left(\left(u_{x}\right)_{j}^{n}\right)^{2}\right], \\
& \mathcal{J}_{3}=h \sum_{j=0}^{2 N}\left[\left(u_{j}^{n}\right)^{4}-6 \beta\left(\left(u_{x}\right)_{j}^{n}\right)^{2}\right] .
\end{aligned}
$$

In Table 3, we presented that the discrete mass, momentum and energy of the numerical solutions for the MRLW equation. From both tables, it is obvious that the proposed scheme is satisfactorily conservative.

\subsection{Interaction of two GRLW solitary waves}

Next, we study the interaction of two well-separated solitary waves having different amplitudes and traveling in the same direction. The initial condition is given by

$$
u_{0}(x)=\sum_{i=1}^{2} A_{i} \operatorname{sech}^{\frac{2}{p-1}}\left[K_{i}\left(x+x_{i}\right)\right]
$$

For $p=2$, we choose $c_{1}=1.3, c_{2}=1.8, x_{1}=15, x_{2}=35, \Delta t=0.1, N=128$, and $\alpha, \beta$ are defined as in Section 5.1. Figure 3 and Figure 4 show the interaction of two solitary solutions 

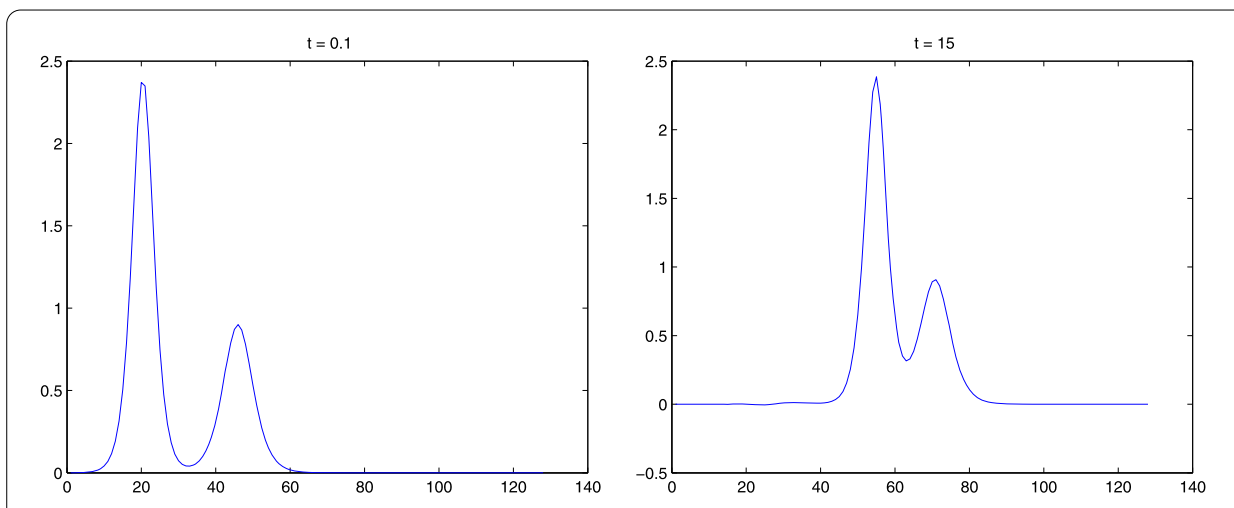

Figure 3 Interaction of two solitary waves for the GRLW equation with $p=2$ at $t=0.1$ and $t=15$, respectively.
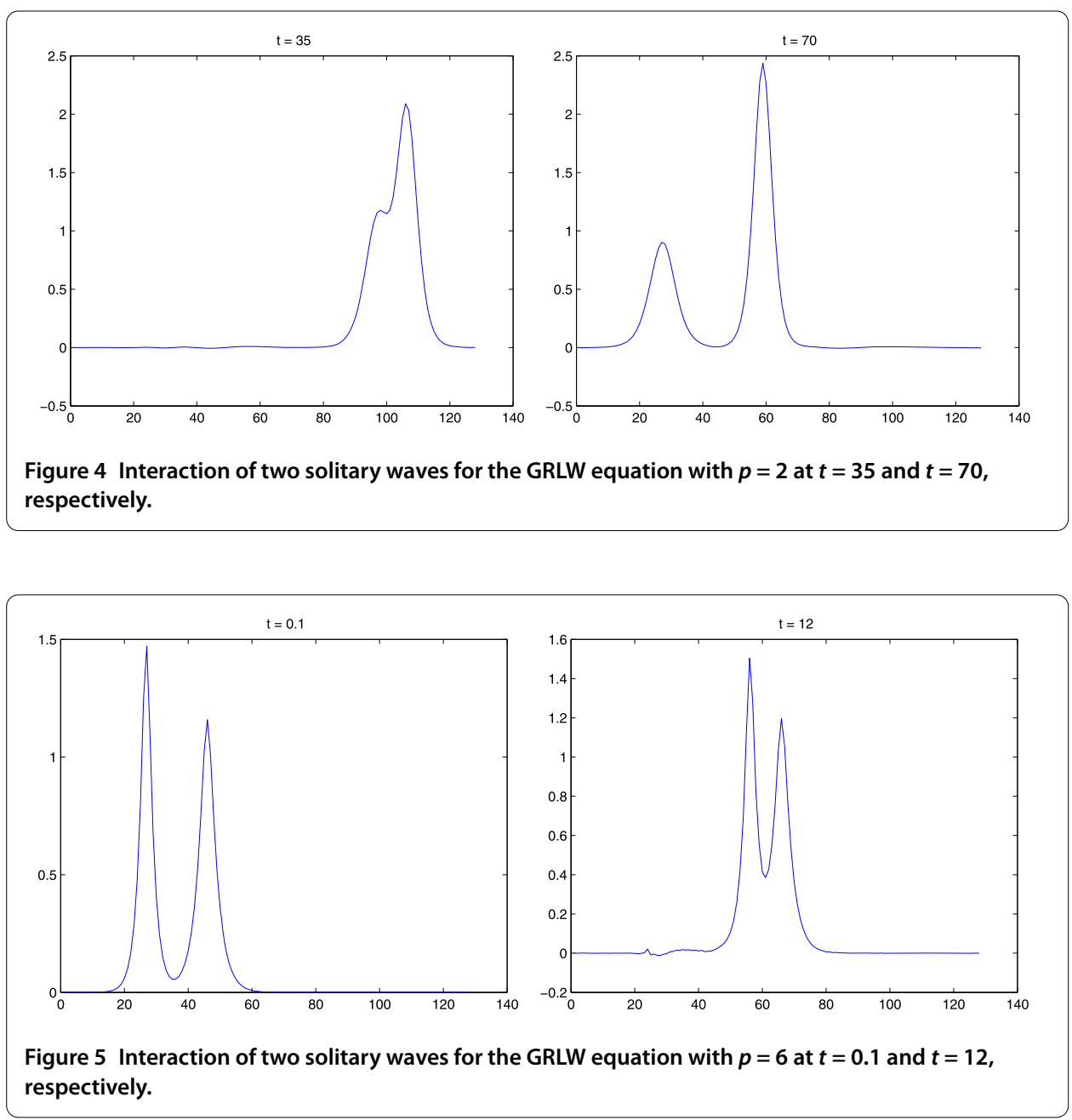

of the GRLW equation. Meanwhile, we briefly plot two figures to show the simulation for $p=6$ at $t=0.1$ and $t=12$ in Figure 5.

From these figures, it can be seen that an oscillation of small amplitude, trailing behind the solitary waves, was observed. This is in accordance with the observations of Soli- 
man [5]. Moreover, the solitary-wave interaction in Figure 5 for $p=6$ shows clearly some evidence of an additional disturbance, which may be due to the fact that the GRLW equation are not completely integrable.

\subsection{Unstable solitary-wave solution}

Souganidis and Strauss [19] derived the following result for the GRLW equation:

(a) if $p<5$, then $u(x, t)$ is $H^{1}$-stable;

(b) if $p>5$, then $u(x, t)$ is $H^{1}$-stable for $c>c_{p}$, and $H^{1}$-unstable for $1 \leq c \leq c_{p}$, where

$$
c_{p}=\frac{1+\sqrt{2-\sigma^{-1}+2 \sigma^{-1}}}{2(\sigma+1)}
$$

and $\sigma=\frac{2}{p-1}$. Note that $c_{p}=1$ when $p=5$ and $c_{p}>1$ for $p \geq 5$. Certainly, for $p=8$, the critical speed is $c_{p}=1.300914$.

Similar to the experiments in [20], the high accuracy scheme we proposed also can simulate this phenomenon, that is, the evolution of solutions emanating from initial data which are perturbations of exact analytical solutions of (1.1) with $p>5$ and $1 \leq c \leq c_{p}$ are featured, where the initial data is a perturbed solitary wave,

$$
\tilde{u}=\gamma u_{0}(x)
$$

and $\gamma$ is called the perturbation parameter; it is used to effect an amplitude perturbation. According to [19], when $\gamma \geq 1$, the solution emanating from the initial data will resolve itself into one or more solitary waves, sometimes accompanied by additional structure. Therefore, mainly attention will be given to evolutions starting with $\tilde{u}$ for $\gamma>1$.

Since unstable solutions have a speed $c$ in the range $1 \leq c \leq c_{p}$ and $c_{p}$ is near 1 , we simulate only one of long-time outcomes for perturbed unstable solitary-wave solutions, with $\alpha=\beta=1, p=8, c=1.01$. To achieve such an outcome, the initial unstable solitary data had to be given enough energy to produce two stable solitary waves. Hence, we choose $\gamma=1.5$. As seen in Figure 6 and Figure 7, the outcomes illustrate how such a process occurred in the experiment.

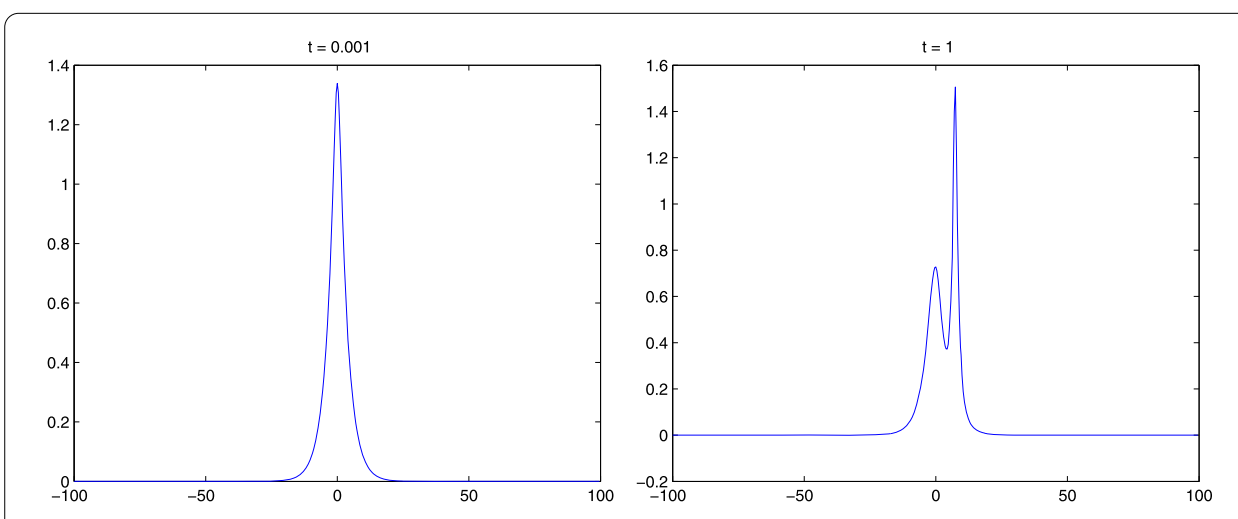

Figure 6 Evolution of an unstable perturbed solitary wave with $c=1.01$ and $\gamma=1.5$ for $p=8$ (I). 

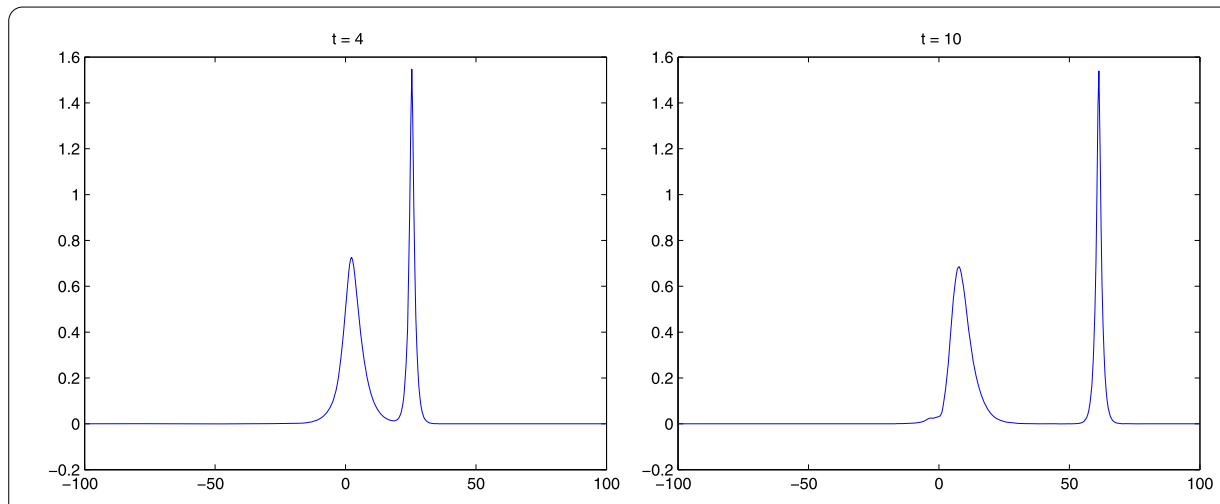

Figure 7 Evolution of an unstable perturbed solitary wave with $c=1.01$ and $\gamma=1.5$ for $p=8$ (II).

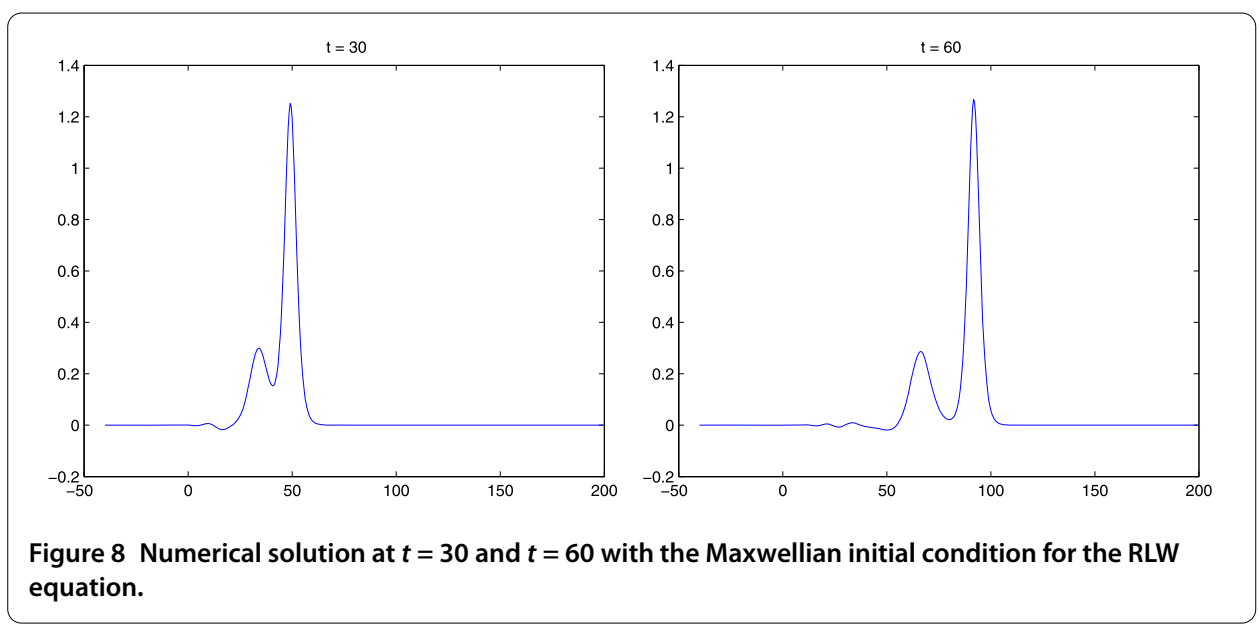

\subsection{Maxwellian initial condition}

A more interesting test case for the GRLW equation is to investigate the long-time asymptotic properties by initiating the wave motion with a Gaussian pulse, namely, the so-called Maxwellian initial condition [34]. We take $\alpha=\frac{1}{2}, \beta=1$ for RLW equation $(p=2)$ and $\alpha=\frac{1}{3}$, $\beta=1$ for MRLW equation $(p=3)$, respectively, with initial data

$$
u(x, 0)=\exp \left(-0.02(x-5)^{2}\right)
$$

In a numerical test, let $N=256, \Delta t=0.01, x_{L}=-40$, and $x_{R}=200$. The numerical solution at different times for both cases are drawn in Figure 8 and Figure 9 for the RLW equation, and Figure 10 and Figure 11 for the MRLW equation, respectively. Furthermore, the quantities for three conservative laws are presented in Table 4. As seen from these results, an initial date with Maxwellian disturbance will resolve into a sequence of solitary waves in the stable range ordered by amplitude with the larger waves in the front, followed by a dispersive tail. Clearly, the presented results are consistent with earlier work on this topic in $[34,35]$.

\section{Conclusion}

In this paper, we propose a fully discrete Fourier pseudospectral scheme for the GRLW equation with the second-order in time accuracy. The theoretical analysis, such as con- 

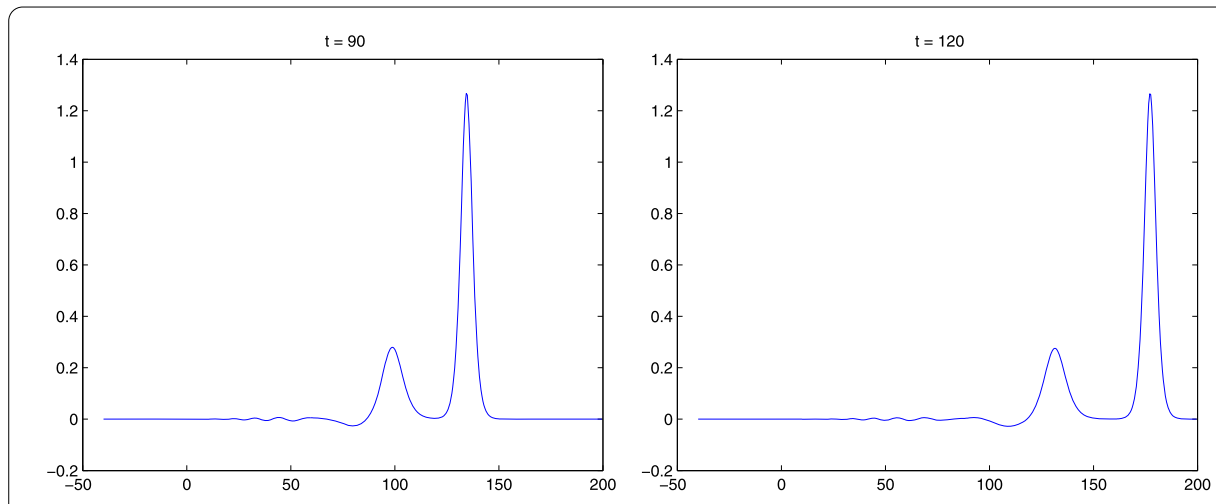

Figure 9 Numerical solution at $t=90$ and $t=120$ with the Maxwellian initial condition for the RLW equation.
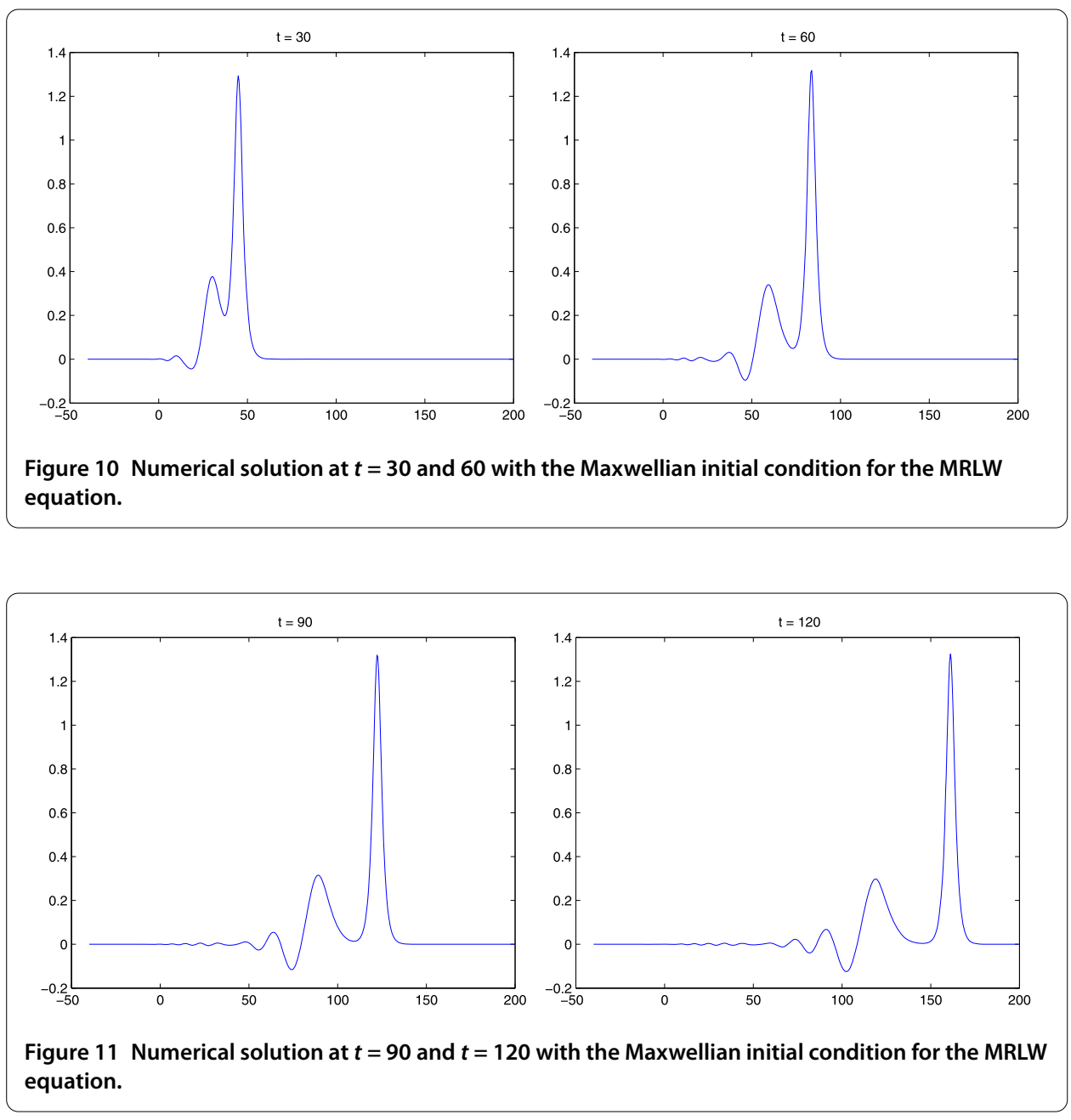

sistence analysis, stability and convergence analysis are presented in detail. Due to the linearized semi-implicit property, the algorithm can be implemented more effectively. Numerical experiments also verifies the second-order accuracy in time and the spectral accuracy in space. Furthermore, thanks to the high accuracy scheme, the evolution of the 
Table 4 Invariants for single soliton of MRLW equation with $N=128$ and $c=\frac{4}{3}$

\begin{tabular}{llllllll}
\hline Time & \multicolumn{3}{l}{ RLW equation $(\boldsymbol{p}=\mathbf{2})$} & & \multicolumn{3}{c}{ MRLW equation $(\boldsymbol{p}=\mathbf{3})$} \\
\cline { 2 - 4 } & $\mathcal{I}_{\mathbf{1}}$ & $\mathcal{I}_{\mathbf{2}}$ & $\mathcal{I}_{\mathbf{3}}$ & & $\mathcal{J}_{\mathbf{1}}$ & $\mathcal{J}_{\mathbf{2}}$ & $\mathcal{J}_{\mathbf{3}}$ \\
\hline$T=0$ & 12.533141 & 9.0394281 & 33.822820 & & 12.533141 & 9.0394281 & 5.2036174 \\
$T=2$ & 12.533141 & 9.0394599 & 33.823003 & & 12.533141 & 9.0394430 & 5.2037832 \\
$T=4$ & 12.533141 & 9.0394331 & 33.823012 & & 12.533141 & 9.0393901 & 5.2041213 \\
$T=6$ & 12.533141 & 9.0393867 & 33.823027 & & 12.533141 & 9.0392994 & 5.2046997 \\
$T=8$ & 12.533141 & 9.0393237 & 33.823048 & & 12.533141 & 9.0391822 & 5.2054479 \\
$T=10$ & 12.533141 & 9.0392517 & 33.823072 & & 12.533141 & 9.0390559 & 5.2062565 \\
\hline
\end{tabular}

unstable solitary wave of the GRLW equation with $p \geq 5$ also be simulated, as established in [19].

\section{Competing interests}

The authors declare that they have no competing interests.

\section{Authors' contributions}

All authors conceived of the study, participated in its design and coordination, drafted the manuscript, participated in the sequence alignment, and read and approved the final manuscript.

\section{Author details}

'School of Science, Southwest University of Science and Technology, Mianyang, 621010, P.R. China. ${ }^{2}$ School of Business, Sichuan University, Chengdu, 610064, P.R. China.

\section{Acknowledgements}

The authors are very grateful to both reviewers for carefully reading this paper and their comments, and thank Dr. Cheng Wang (University of Massachusetts, Dartmouth) for his valuable discussion. This work is supported by the Applied Basic Research Project of Sichuan Province (No. 2013JY0096) and the National Natural Science Foundation of China (No. 11202175)

Received: 16 May 2015 Accepted: 22 October 2015 Published online: 29 October 2015

\section{References}

1. Peregrine, DH: Long waves on beach. J. Fluid Mech. 27(4), 815-827 (1967)

2. Benjamin, TB, Bona, JL, Mahony, JJ: Model equations for long waves in nonlinear dispersive systems. Philos. Trans. R. Soc. Lond. A 272(1220), 47-78 (1972)

3. Miller, JR, Weinstein, Ml: Asymptotic stability of solitary waves for the regularized long-wave equation. Commun. Pure Appl. Math. 49(4), 399-441 (1996)

4. Wazwaz, AM: Analytic study on nonlinear variants of the RLW and the PHI-four equations. Commun. Nonlinear Sci. Numer. Simul. 12(3), 314-327 (2007)

5. Soliman, AA: Numerical simulation of the generalized regularized long wave equation by He's variational iteration method. Math. Comput. Simul. 70(2), 119-124 (2005)

6. Zhang, L: A finite difference scheme for generalized regularized long-wave equation. Appl. Math. Comput. 168(2), 962-972 (2005)

7. Chang, Q, Wang, G, Guo, B: Conservative scheme for a model of nonlinear dispersive waves and its solitary waves induced by boundary motion. J. Comput. Phys. 93(2), 360-375 (1991)

8. Zheng, K, Hu, J: High-order conservative Crank-Nicolson scheme for regularized long wave equation. Adv. Differ. Equ. 2013, Article ID 287 (2013)

9. $\mathrm{Hu}, \mathrm{J}$, Zheng, K, Zheng, M: Numerical simulation and convergence analysis of a high-order conservative difference scheme for SRLW equation. Appl. Math. Model. 38(23), 5573-5581 (2014)

10. Akbari, R, Mokhtari, R: A new compact finite difference method for solving the generalized long wave equation. Numer. Funct. Anal. Optim. 35(2), 133-152 (2014)

11. Sloan, DM: Fourier pseudospectral solution of the regularized long wave equation. J. Comput. Appl. Math. 36(2), 159-179 (1991)

12. Djidjeli, K, Price, WG, Twizell, EH, Cao, Q: A linearized implicit pseudo-spectral method for some model equations: the regularized long wave equations. Commun. Numer. Methods Eng. 19(11), 847-863 (2003)

13. Roshan, T: A Petrov-Galerkin method for solving the generalized regularized long wave (GRLW) equation. Comput. Math. Appl. 63(5), 943-956 (2012)

14. Achouri, T, Ayadi, M, Omrani, K: A fully Galerkin method for the damped generalized regularized long-wave (DGRLW) equation. Numer. Methods Partial Differ. Equ. 25(3), 668-684 (2009)

15. Achouri, T, Omrani, K: Numerical solutions for the damped generalized regularized long-wave equation with a variable coefficient by Adomian decomposition method. Commun. Nonlinear Sci. Numer. Simul. 14(5), 2025-2033 (2009)

16. Kaya, D: A numerical simulation of solitary-wave solutions of the generalized regularized long-wave equation. Appl. Math. Comput. 149(3), 833-841 (2004) 
17. Mokhtari, R, Mohammadi, M: Numerical solution of GRLW equation using Sinc-collocation method. Comput. Phys. Commun. 181(7), 1266-1274 (2010)

18. Mohammadi, M, Mokhtari, R: Solving the generalized regularized long wave equation on the basis of a reproducing kernel space. J. Comput. Appl. Math. 235(14), 4003-4014 (2011)

19. Souganidis, PE, Strauss, WA: Instability of a class of dispersive solitary waves. Proc. R. Soc. Edinb. A 114, 195-212 (1990)

20. Bona, JL, McKinney, WR, Restrepo, JM: Stable and unstable solitary-wave solutions of the generalized regularized long-wave equation. J. Nonlinear Sci. 10(6), 603-638 (2000)

21. Durán, A, López-Marcos, MA: Numerical behaviour of stable and unstable solitary waves. Appl. Numer. Math. 42(1), 95-116 (2002)

22. Boyd, J: Chebyshev and Fourier Spectral Methods, 2nd edn. Dover, New York (2001)

23. Hesthaven, J, Gottlieb, S, Gottlieb, D: Spectral Methods for Time-Dependent Problems. Cambridge University Press, Cambridge (2007)

24. Maday, Y, Quarteroni, A: Spectral and pseudospectral approximation to Navier-Stokes equations. SIAM J. Numer. Anal. 19(4), $761-780$ (1982)

25. Tadmor, E: Convergence of spectral methods to nonlinear conservation laws. SIAM J. Numer. Anal. 26(1), 30-44 (1989)

26. Tadmor, E: The exponential accuracy of Fourier and Chebyshev differencing methods. SIAM J. Numer. Anal. 23(1), 1-10 (1986)

27. Weinan, E: Convergence of Fourier methods for Navier-Stokes equations. SIAM J. Numer. Anal. 30(3), 650-674 (1993)

28. Guo, BY, Zou, J: Fourier spectral projection method and nonlinear convergence analysis for Navier-Stokes equations. J. Math. Anal. Appl. 282(2), 766-791 (2003)

29. Gottlieb, S, Wang, C: Stability and convergence analysis of fully discrete Fourier collocation spectral method for 3-D viscous Burgers' Equation. J. Sci. Comput. 53(1), 102-128 (2012)

30. Cheng, K, Feng, W, Gottlieb, S, Wang, C: A Fourier pseudospectral method for the 'good' Boussinesq equation with second-order temporal accuracy. Numer. Methods Partial Differ. Equ. 31(1), 202-224 (2015)

31. Du, Q, Guo, B, Shen, J: Fourier spectral approximation to a dissipative system modeling the flow of liquid crystals. SIAM J. Numer. Anal. 39(3), 735-762 (2001)

32. Canuto, C, Quarteroni, A: Approximation results for orthogonal polynomials in Sobolev spaces. Math. Comput. 38(157), 67-86 (1982)

33. Baskara, A, Lowengrub, JS, Wang, C, Wise, SM: Convergence analysis of a second order convex splitting scheme for the modified phase field crystal equation. SIAM J. Numer. Anal. 51(5), 2851-2873 (2013)

34. Mokhtari, R, Ziaratgahi, ST: Numerical solution of RLW equation using integrated radial basis functions. Appl. Comput. Math. 10(3), 428-448 (2011)

35. Chegini, NG, Salaripanah, A, Mokhtari, R, Isvand, D: Numerical solution of the regularized long wave equation using nonpolynomial splines. Nonlinear Dyn. 69(1-2), 459-471 (2012)

\section{Submit your manuscript to a SpringerOpen ${ }^{\ominus}$ journal and benefit from:}

- Convenient online submission

Rigorous peer review

- Immediate publication on acceptance

- Open access: articles freely available online

- High visibility within the field

- Retaining the copyright to your article 